\title{
Synthetic studies related to oral $\beta$-lactam antibiotics
}

\author{
Mitsuru Yoshioka \\ Shionogi Research Laboratories, Shionogi \& Co., Ltd., \\ Fukushima-ku, Osaka 553, Japan
}

\begin{abstract}
Development of an industrial process for producing 7432-S, a new oral cephem antibiotic, is outlined. The required components, 2-(2benzyloxycarbonylamino-4-thiazoly 1 )-4-(3-methylbut-2-enyloxycarbony 1 )but2-enoic acid (2b) and diphenylmethyl 7B-amino-3-cephem-4-carboxylate (3b) (or its precursor $3^{\prime}$ ), were prepared starting from methyl 2-(2-amino-4thiazolyl)acetate $(12)$ and penicill in $G$, respectively. Condensation of the two components followed by deprotection (after reduction or el imination) and epimerization-crystallization gave $7432-5$ in $36-42 \%$ overall yields from penicillin G. Special efforts have been made to utilize inexpensive and non-hazardous materials and to maximize one-pot procedures.
\end{abstract}

\section{INTRODUCTION}

$3^{\prime}$-Norcephalosporins $\left(1: R^{1}=H\right.$ or a non-carbon substituent) are an important class of heteroatom antibiotics which have been widely used in the treatment of a variety of infections. Since the $3^{\prime}$-nor nuclei are not naturally occurring, they should be derived from penicillins or cephalosporins, commercially available fermentation products (ref. 1). Recently we at Shionogi have been developing 7432-S, a new oral 3'-norcephalosporin exhibiting a potent activity against most gram-negative and some gram-positive bacteria, with a very excellent oral bioavailability (ref. 2), and have needed to explore an industrial synthetic route to this antibiotic (Fig. 1). This paper outlines our efforts to develop an industrially feasible, economical process for producing 7432-S, starting from penicillin $G$, the least expensive $\beta$-lactam.

Oral 3'-Norcephalosporins

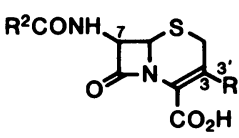

*: D-form
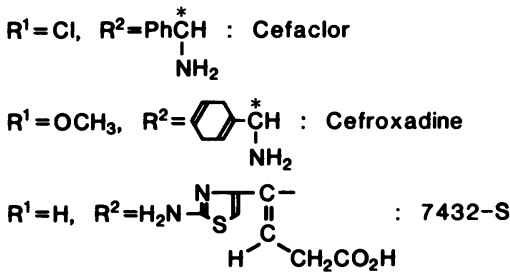

Parenteral 3'-Norcephalosporins

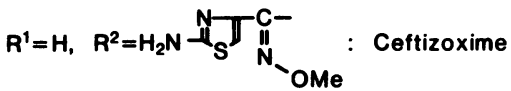

Fig. 1. 3'-Norcephalosporins

\section{SYNTHETIC ROUTE}

7432-S can be produced by condensing a 7-side chain acid 2 with a 78 -aminocephem nucleus $\underline{3}$ or its precursor $\underline{3}^{\prime}$, followed by elimination or reduction when $\underline{3}^{\prime}$ is used, deprotection, epimerization and finally crystallization (Fig. 2). Thus, the keys for successful largescale production of 7432-S would be economical preparation of these components 2 and $\underline{3}$ or $3^{\prime}$ as well as efficient epimerization-crystallization to yield only $7432-S[(Z)-i$ somer $]$ from a mixture with its (E)-isomer, with taking into account the following crucial factors for industrialization:

1. Use of inexpensive starting materials.

2. Use of inexpensive and non-hazardous reagents and solvents.

3. Minimization of separation-purification procedures by improving step yields and employing one-pot procedures as much as possible.

4. Facile scale-up; no column-chromatographic separation. 

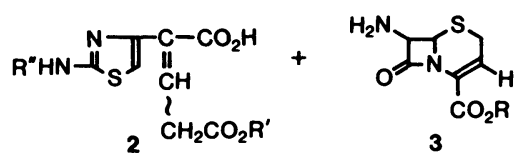<smiles>[R]OC1=C([N+](=O)[O-])N2C(=O)C(N)C2SC1</smiles>

3 $3^{\prime}$

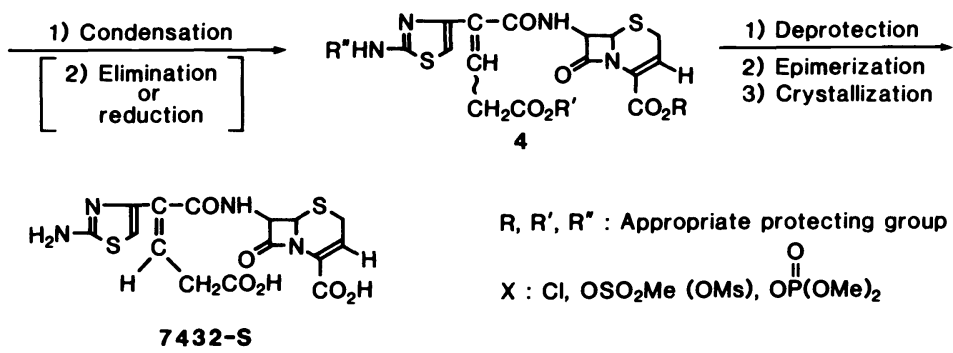

Fig. 2. Synthetic Route to $\mathbf{7 4 3 2 - S}$

\section{PREPARATION OF 7-SIDE CHAIN ACID 2}

The synthetic route applied to earlier preparation of acid 2 a consists of facile conversion of diketene into diphenylmethyl 2-(2-amino-4-thiazoly1)acetate (6), amine protection followed by formylation to give enol 7, Wittig reaction affording diester 8 a and partial deprotection of the diphenylmethyl ester to yield the objective acid $2 a$. This route has the following problems (rectangles in Fig. 3) that make the scale-up difficult:

1. The formylation using sodium hydride was exothermic and difficult to control on large scales.

2. In the Wittig reaction, the phosphorane reagent was considerably expensive and impractical chromatographic separation of triphenylphosphine oxide was necessary.

3. Partial deprotection needed hazardous trifluoroacetic acid.

4. The benzyl group in acid 2 a could not be cleanly deblocked in the final deprotection step giving $7432-S$.

Although this route could be scaled up in pilot plants by modifications to solve the third and fourth problems (replacement of $\mathrm{CHPh}_{2}$ by $\mathrm{Me}$ and $\mathrm{CH}_{2} \mathrm{Ph}$ by $\mathrm{t}-\mathrm{Bu}$ in $8 \mathrm{a}$; then partial hydrolys is of the methyl ester), the first and second ones remained unsolved despite our various attempts.
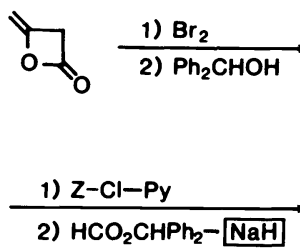<smiles>O=C(CBr)COC(=O)c1ccccc1</smiles>
1) $\left(\mathrm{H}_{2} \mathrm{~N}\right)_{2} \mathrm{C}=\mathrm{S}$ 2) $\mathrm{K}_{2} \mathrm{CO}_{3}$ $\mathrm{H}-\mathrm{AMT}-\mathrm{CH}_{2} \mathrm{CO}_{2} \mathrm{CHPh}_{2}$

$6(81 \%)$

$$
7 \text { (70\%) }
$$

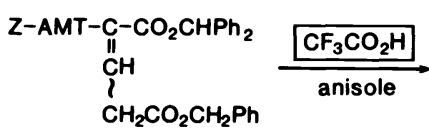

8a (87\%)<smiles></smiles>

2a $(74 \%)$

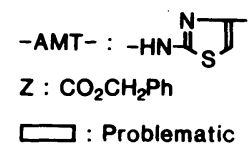

Fig. 3. Early Synthesis of 7-Side Chain Acid 2a

Thus, extensive studies were initiated to search for more practical synthesis of the 7-side chain acid 2. Soon an attractive route (Fig. 4) has been found which consists of conversion of the acetonedicarboxylate-magnesium complex 9 into aminothiazolyl enol 10, borohydride reduction to alcohol 11 and the three-step transformation into diester $8 \mathrm{~b}$ that can be derived to acid 2 as discussed later. This route has been given up without making any attempts to improve the low yield (59\%) of enol 10, when dimethyl 1,3-acetonedicarboxylate turned out to be considerably expensive.

After many unsuccessful attempts, we could finally reach to an industrially feasible fourstep process for producing acid 2 starting from methyl 2-(2-amino-4-thiazoly1)acetate (12), an inexpensive, abundant material (Fig. 5). Acylation of 12 with benzyl chloroformate 

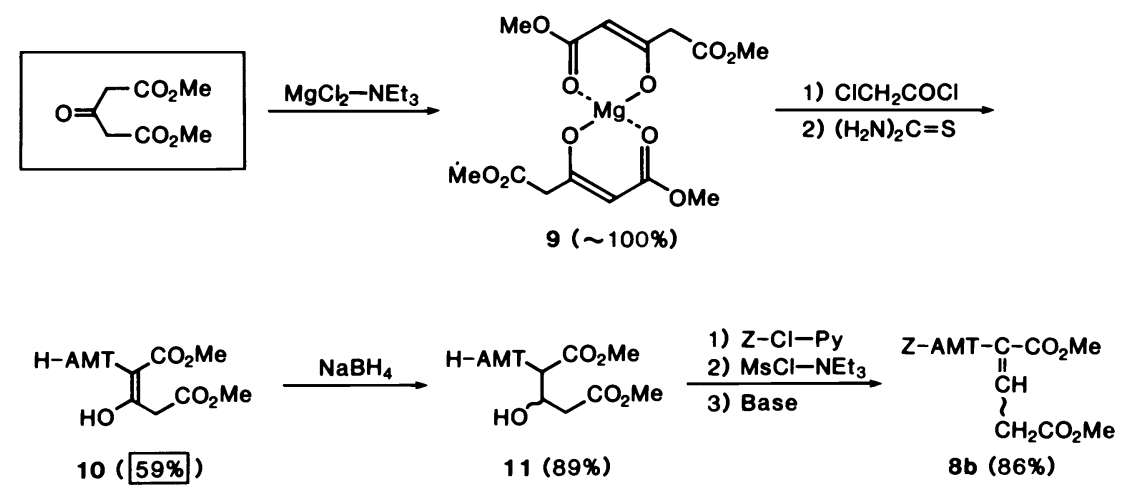

Fig. 4. Improved Synthesis of 7-Side Chain Intermediate $\mathbf{8 b}$

(Z-Cl) proceeded quite satisfactorily under the Schotten-Baumann conditions to give protected ester $13 \mathrm{~b}$ which was condensed with methyl $\beta$-methoxyacrylate to afford diester $8 \mathrm{~b}$. Unexpectedly, similar Michael condensation of ester $13 \mathrm{c}$ with $\beta$-chloro- or mesyloxyacrylate did not yield diester $8 c$ in our earlier attempts to delay the use of $\beta$-methoxyacrylate, a seemingly less-reactive Michael acceptor. Diester $8 \mathrm{~b}$ obtained as a toluene extract was easily hydrolyzed with aqueous alkali in a two-phase system to give diacid 14 which could be partially esterified to half ester 2 with an alcohol in the presence of an acid catalyst. In this tricky procedure, steric hindrance and relative carboxyl basicity seem to be important factors for selective monoesterification and only primary alcohols or some (less-hindered) secondary alcohols catalyzed by a strong acid can smoothly attack the primary carboxylic acid in a regiospecific manner leading to half ester 2 . Thus, tert-butyl alcohol and diphenylmethanol, favorable protecting alcohols, can not be used and protecting primary alcohols other than problematic benzyl alcohol have been sought. Para-methoxybenzyl alcohol and 3-methyl-2-buten-1-01 (prenyl alcohol) were found satisfactory, of which the latter was selected from its lower price. Thus, the required 7-side chain component $2 \mathrm{~b}$ has been produced in $71 \%$ overall yield on pilot-plant scales. Incidentally, an attempt failed to obtain the half ester 2 a by direct Michael condensation of $\beta$-alkoxyacrylate with a dianion of thiazolylacetic acid $\underline{15}$.

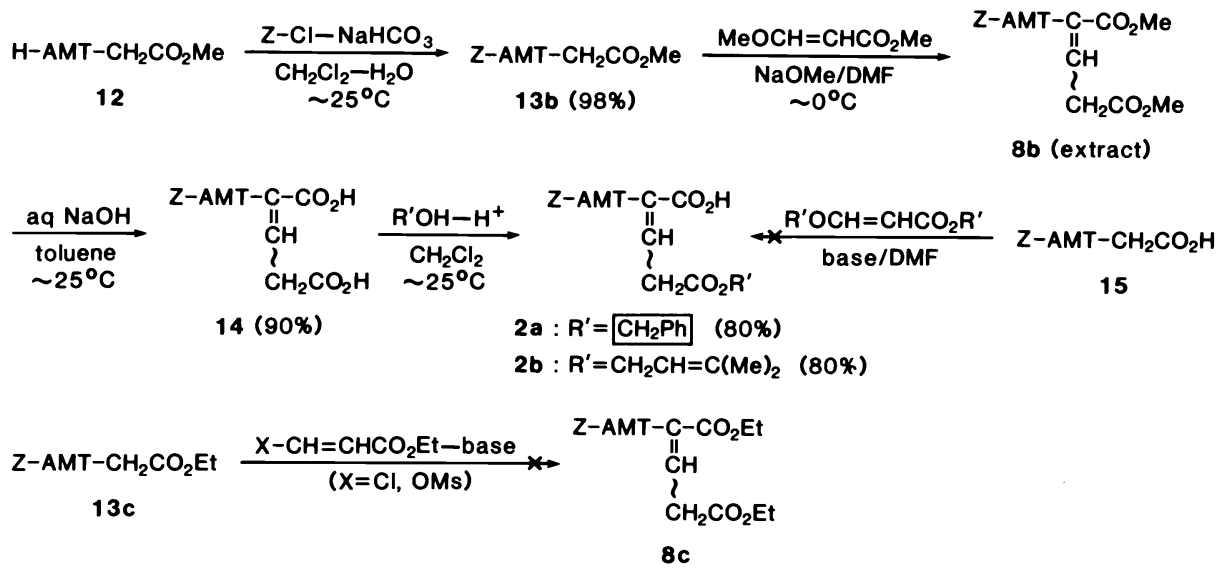

Fig. 5. Industrial Synthesis of 7-Side Chain Acid 2b

\section{PREPARATION OF 7 $\beta$-AMINOCEPHEM NUCLEI}

For synthesis of the 7-amino component 3 or $3^{\prime}$ we have decided to modify our earlier, industrially feasible route (ref. 3 , Fig. 6) involving conversion of inexpensive penicillin $G$ into thiazoline 18 a via S-oxide ester 17 a by known procedures (ref. 4), ozonolysis to enol 19a, and our unique one-pot procedure leading to 3-hydroxycephem intermediate $24 a$ via mesylate $20 \mathrm{a}$, enamine bromide $22 \mathrm{a}$, and thiol $23 \mathrm{a}$. The problems to be solved in this wellstudied route are indicated as follows:

1. The use of peracetic acid that should be stored with care in an isolated, air-conditioned warehouse.

2. The use of highly mutagenic p-nitrobenzyl bromide.

3. The use of volatile, stinking dimethyl sulfide (bp $36^{\circ} \mathrm{C}$ ).

4. Formation of C-mesylate 21 lowering the product yield. 

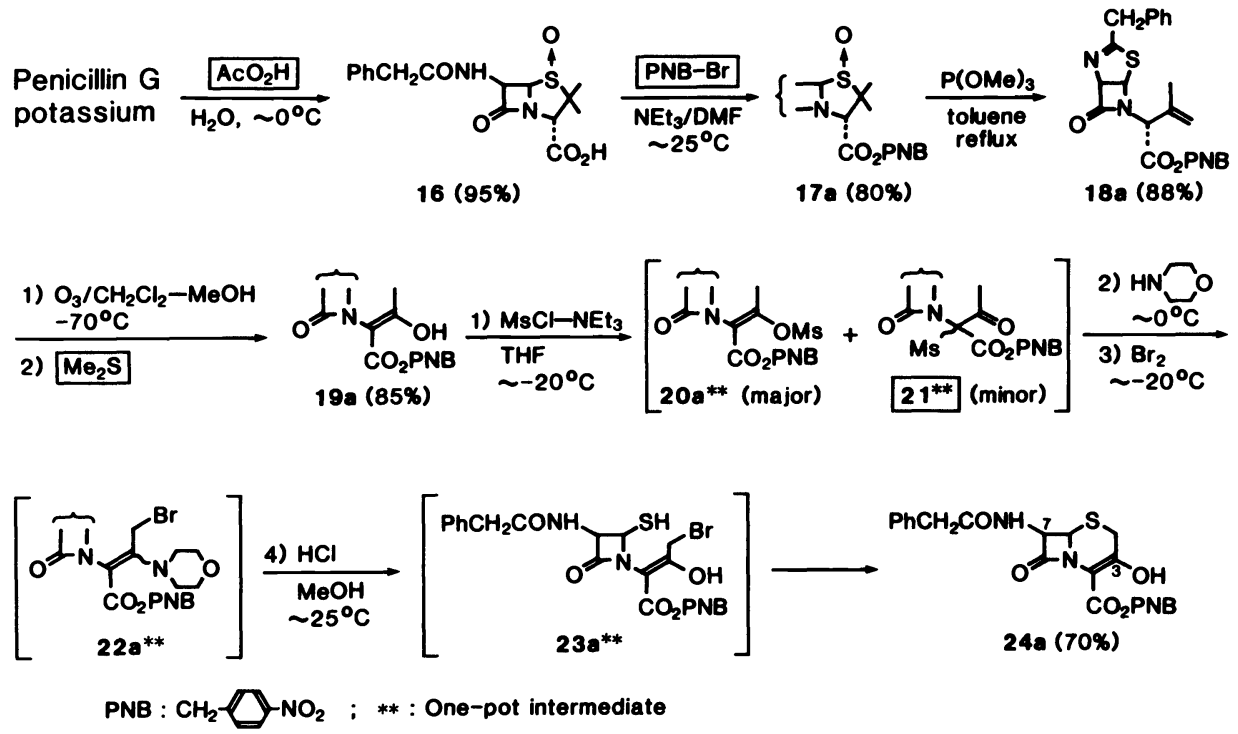

Fig. 6. Early Synthesis of $3^{\prime}$-Norcephalosporin Intermediate $24 a$

In our improved industrial process, penicillin $G$ diphenylmethyl ester obtained as an extract was oxidized with hydrogen peroxide in the presence of formic acid and polyphosphoric acid (probably assisting the formation of performic acid, ref. 5) to give S-oxide ester 17b in excellent yield, which was converted into enol 19b by ozonolysis of thiazoline $18 \mathrm{~b}$ with substituting trimethyl phosphite for low-boiling dimethyl sulfide. When methanesulfonyl chloride (MSCl) was replaced by p-toluenesulfonyl chloride (TsCl) in our one-pot process, the C-sulfonyl by-product like 21 was not formed and 3-hydroxycephem intermediate $24 \mathrm{~b}$ obtained in $63 \%$ overall yield on pilot-plant scales, where only three crystallization-isolation procedures were necessary (Fig. 7).
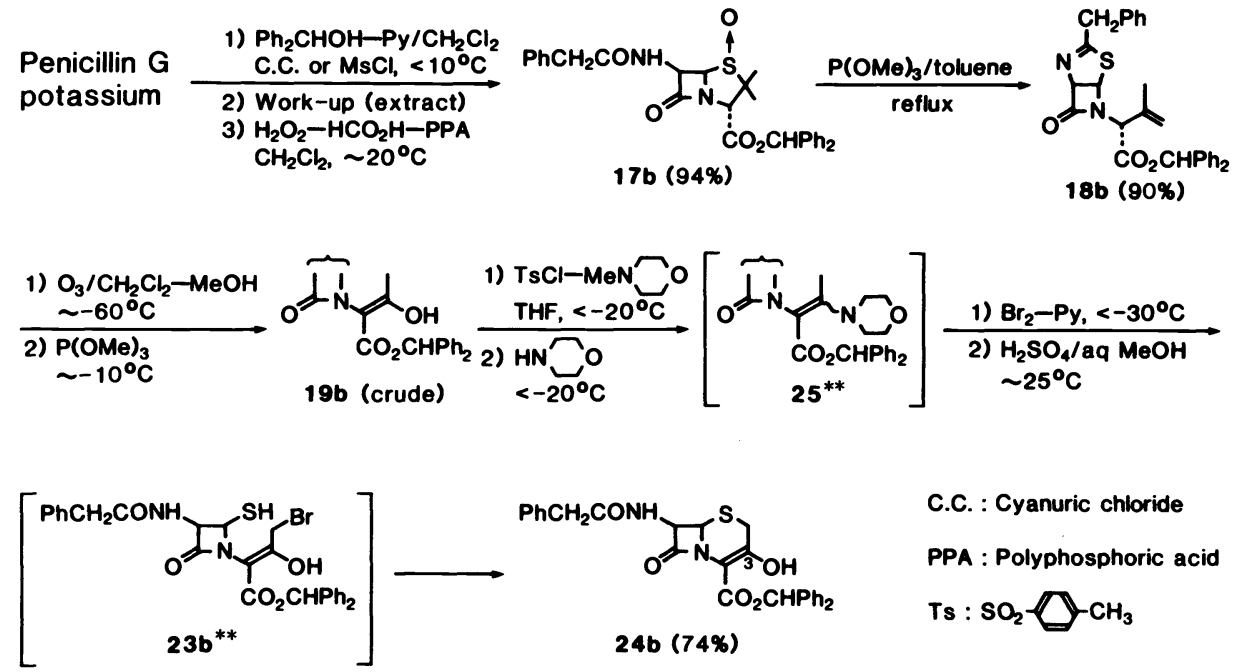

Fig. 7. Improved Industrial Process for Synthesis of $3^{\prime}$-Nor Intermediate 24b

Two routes have been still competing for transforming this intermediate $24 \mathrm{~b}$ into the desired 7-amino nuclei 3 and $3^{\prime}$ (Fig. 8). One route consists of smooth mesylation of $24 \mathrm{~b}$ to enol mesylate 26 and the side-chain cleavage by the well-established two-step, onepot procedure giving 3-mesyloxy-7 - -aminocephem 3 'b, which can be used as the acylation substrate with or without further zinc-acid reduction to amine $3 b$. Another route involves borohydride reduction of 3-hydroxycephem $24 \mathrm{~b}$ at low temperature affording 3-hydroxycepham 27 and mesylation followed by the side-chain cleavage to give $7 \beta$-aminocepham $3^{\prime} c$, which can be used as the acylation substrate or eliminated with base to amine $3 \mathrm{~b}$. The low temperature (below $-60^{\circ} \mathrm{C}$ ) is crucial for successful hydride reduction to 3 -hydroxycepham 27 (ref. 6), because its yield was greatly reduced with formation of diphenylmethanol and by-product $27^{\prime}$ when the reduction temperature was raised to $0^{\circ} \mathrm{C}$ as exemplified in the literature (ref. 7). Interestingly, the hydride attack occurred at the seemingly more 


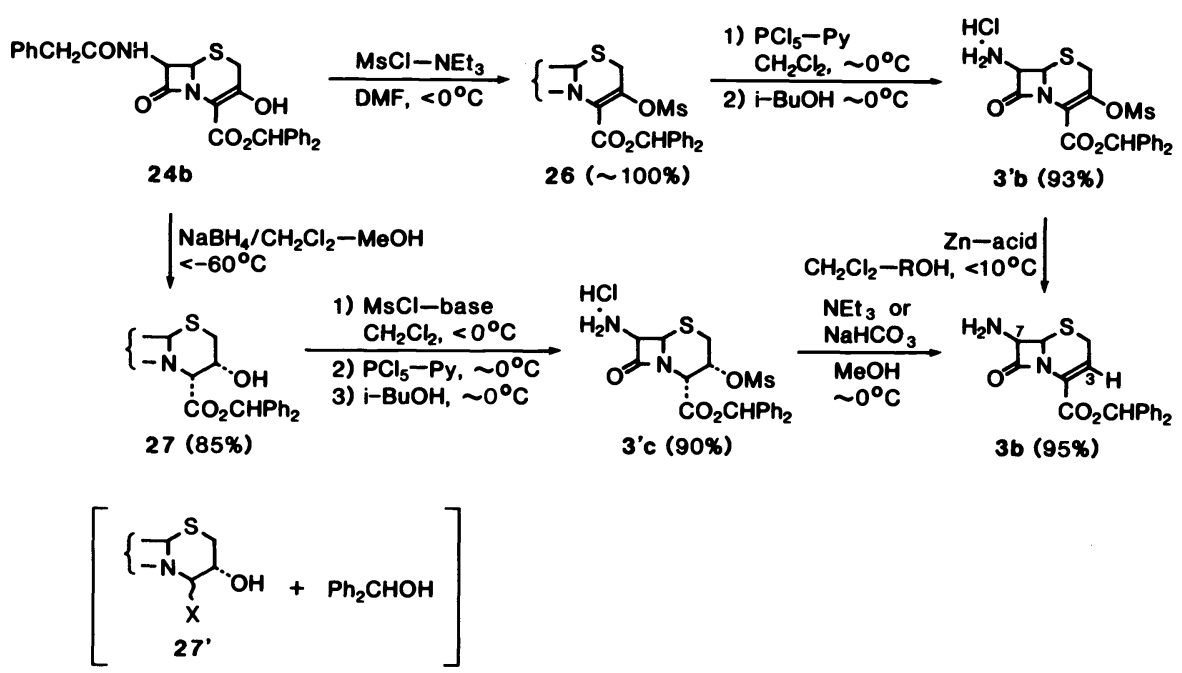

Fig. 8. Synthetic Routes to 7ß-Amino Nuclei 3 \& 3'

hindered $\beta$-side affording $3 \alpha$-hydroxycepham 27 as in the reduction of 3-hydroxy-1-oxa-3cephems (ref. 8). This preferred $\beta$-side attack can be explained by our recently proposed generalization (ref. 9) that the $\beta$ not $\alpha$ side is actually less-hindered in 3-exomethylene cephams (the 3-keto form of enol $24 \mathrm{~b}$ in the present case). With regard to comparison of the two synthetic routes, the former route seems somewhat advantageous at present, but the final selection will be made after comparing these routes including the choice of the acylation substrate on pilot-plant scales.

\section{PRODUCTION OF 7432-S}

Acylation of the $7 \beta$-amino nuclei $3 b, 3^{\prime} b$, and $3^{\prime} c$ with the side chain acid $2 b$ could be effected by the usual methods employing mesyl chloride, phosphorus oxychloride, thionyl chloride etc as the condensing agent in the presence of a base such as triethylamine or pyridine. Diester $4 \mathrm{~b}$ containing the $(\underline{E})$-isomer predominantly was obtained in excellent yield, after zinc-acid reduction or base el imination when precursor $3^{\prime} b$ or $3^{\prime} c$ was used. The three protecting groups in diester $4 \mathrm{~b}$ could be smoothly deblocked at once by our practical, aluminum trichloride-anisole procedure (ref. 10) (Fig. 9). However, isolation of 7432-S from the reaction mixture was not straightforward, because amino acids, 7432-S and its (E)-isomer, could not be extracted with an appropriate solvent or precipitated out from the acid-treatment mixture containing a large amount of aluminum trichloride.
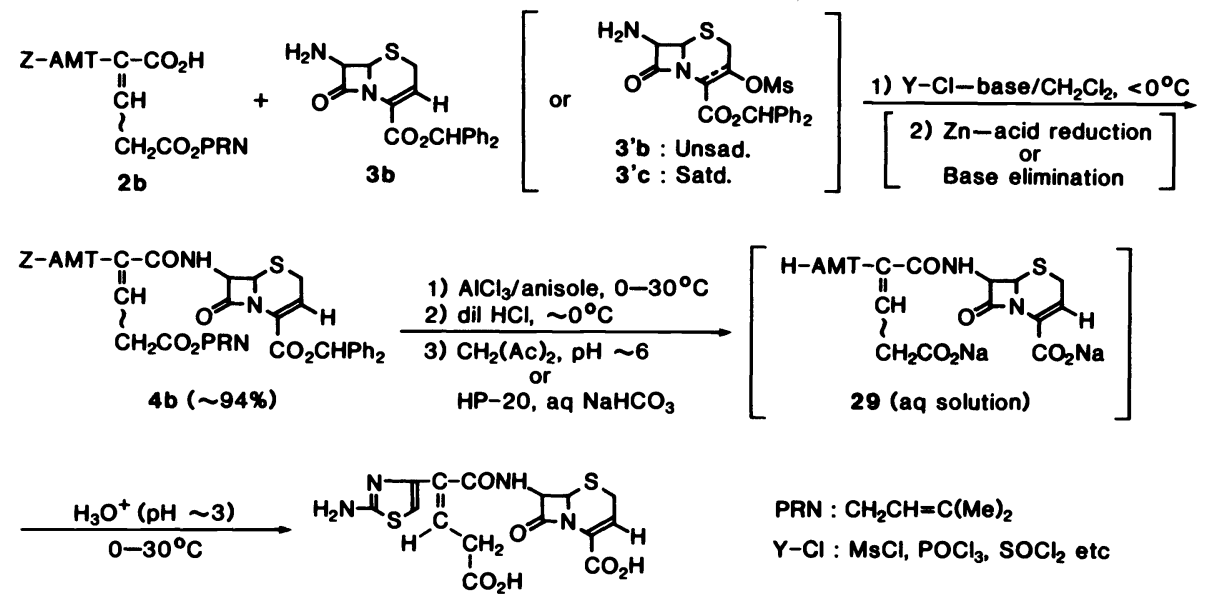

7432-S (80\%; $36-42 \%$ overall yield from penicillin G)

Fig. 9. Production of 7432-S 
First, a procedure utilizing a big column of HP-20 $0^{\circledR}$, a high porous polymer supplied by Mitsubishi Chemical Industries, has been developed to adsorb the amino acid product which could be eluted with dilute alkali, after washing out aluminum trichloride with water, to yield disodium salt 29 . The dilute eluates containing 29 were concentrated by using a reverse osmosis membrane such as NTR-7250-S4 (Nitto Electric Industrial Co., Ltd.) and then adjusted to $\mathrm{pH}$ about 3 to precipitate 7432-S as fine crystals. Fortunately, the unwanted (E)-isomer formed as a major product is considerably soluble in the aqueous acid solution where rapid equilibration with the desired $(Z)$-isomer occurs to finally precipitate out only a less-soluble (Z)-isomer, 7432-S. Later, this procedure needing a large equipment was taken over by a precipitation procedure using acetylacetone. In the improved procedure, the acid layer containing aluminum trichloride and the amino acid product was mixed with acetylacetone and then adjusted to $\mathrm{pH}$ about 6 to form the aluminum hydroxide-acetylacetone complex that, unlike aluminum hydroxide itself, could be easily filtered off. The filtrate containing disodium salt 29 was adjusted to pH about 3 to yield $7432-\mathrm{S}$. The overall yields are $36-42 \%$ from penicillin G.

\section{CONCLUSION}

In production of a new oral cephem antibiotic, 7432-S, the required 7-side chain component $2 \mathrm{~b}$ was prepared in $71 \%$ overall yield by a four-step process starting from ester 12 (Fig. 5) and the $7 \beta$-amino nucleus $3 b$ (or its precursor) in $253 \%$ overall yield by a severa 7 -step process starting from penicillin G (Figs. 7 \& 8). 7432-S was then obtained in $275 \%$ yield by condensation of the two components, (reduction or el imination), deprotection, and epimerization-crystallization (Fig. 9). The successful development of this industrial process, utilizing inexpensive and non-hazardous heteroatom materials without any chromatographic separation, not only facilitates industrial production of our new useful oral antibiotic, 7432-S, but also would contribute to production of other $3^{\prime}$-norcephalosporin antibiotics which could be derived from a 3-hydroxycephem intermediate such as $24 \mathrm{~b}$ or a $7 \beta$-aminocephem nucleus such as $3 \mathrm{~b}$.

\section{Acknowledgements}

This paper is a brief account of work conducted by many excellent researchers of Shionogi \& Co., Ltd. for the past three years. The author would like to express his sincere thanks to $\mathrm{Dr}$. T. Komeno, Director of Shionogi Research Laboratories, and Dr. W. Nagata, Director of Shionogi Production Department, for their generous permission to disclose the industrial process for producing 7432-S that otherwise should be kept confidential. The author also is greatly indebted to Drs. Y. Hamashima, T. Tsuji, H. Onoue, Y. Hamada, H. Nishitani, and $Y$. Sendo of our Research Laboratories and Drs. K. Tokuyama, T. Nishino, M. Kiyokawa, F. Takami, and M. Tanaka of our Production Department as well as their coworkers who have taken their earnest and intelligent efforts to improve the procedures outlined here.

\section{REFERENCES}

1. R. Bucourt, Recent Advances in the Chemistry of B-Lactam Antibiotics, p. 1, Royal Society of Chemistry, London (1981).

2. (a) T. Yoshida, Y. Hamashima, S. Matsuura, Y. Komatsu and S. Kuwahara, 26th Intersci. Conf. Antimicrob. Agents Chemother., abstract 589 (1986).

(b) K. Hirano, T. Yoshida, T. Matsubara, K. Mizojiri, F. Kobayashi and S. Kuwahara, ibid, abstract 590 (1986).

(c) M. Nakashima, M. Iida, T. Yoshida, T. Kitagawa, T. Oguma and H. Ishii, ibid, abstract 591 (1986).

3. Y. Hamashima, K. Ishikura, H. Ishitobi, H. Itani, T. Kubota, K. Minami, M. Murakami, W. Nagata, M. Narisada, Y. Nishitani, T. Okada, H. Onoue, H. Satoh, Y. Sendo, T. Tsuji and M. Yoshioka, Recent Advances in the Chemistry of B-Lactam Antibiotics, p. 243, Royal Society of Chemistry, London (1976).

4. R.D.G. Cooper and F.L. José, J. Am. Chem. Soc. 92, 2575 (1970).

5. M. Yoshioka and M. Tanaka, Jpn. Koka $\bar{i}$ Tokkyo Koho, JP 6150,986 (1986).

6. Y. Hamashima and F. Takami, U.S. Patent, to be published (1987).

7. R. Scartazzini and H. Bickel, Helv. Chim. Acta 57, 1919 (1974).

8. T. Aoki, K. Minami, T. Kubota, Y. Hamashima and W. Nagata, Heterocycles 18, 201 (1982).

9. T. Aoki, T. Konoike, H. Itani, T. Tsuji, M. Yoshioka and W. Nagata, Tetrahedron 39 , 2215 (1983).

10. T. Tsuji, T. Kataoka, M. Yoshioka, Y. Sendo, Y. Nishitani, S. Hirai, T. Maeda and W. Nagata, Tetrahedron Lett. 30, 2793 (1979). 